\title{
Establishing Normative Values for the Barnett Balance Assessment-Sit (BBA-SIT)
}

\author{
Lela Rasegan, Holly Klein, Paige Bell, Brandon Bishop, Ellen Herlache-Pretzer \\ Master of Science in Occupational Therapy Program, Saginaw Valley State University, University Center, USA \\ Email: lelongwo@svsu.edu
}

How to cite this paper: Rasegan, L., Klein, H., Bell, P., Bishop, B. and Herlache-Pretzer, E. (2018) Establishing Normative Values for the Barnett Balance Assessment-Sit (BBA-SIT). Open Journal of Therapy and Rehabilitation, 6, 56-64.

https://doi.org/10.4236/ojtr.2018.62005

Received: March 28, 2018

Accepted: May 6, 2018

Published: May 9, 2018

Copyright $\odot 2018$ by authors and Scientific Research Publishing Inc. This work is licensed under the Creative Commons Attribution International License (CC BY 4.0).

http://creativecommons.org/licenses/by/4.0/

\begin{abstract}
The purpose of this descriptive study was to collect quantitative, normative data for the Barnett Balance Assessment-Sitting (BBA-SIT), a newly-developed dynamic sitting balance assessment tool based on the Barnett Balance Assessment (BBA). The BBA-SIT was administered to a total of 180 participants (30 people in each of the following age categories: 18 - 29, 30 - 39, 40 - 49, 50 59, $60-69$, and 70+) who did not have any current balance deficits. A review of normative data collected indicated no variation in the total assessment scores within and between groups. Future research on the BBA-SIT is needed with balance-impaired populations to determine if the BBA-SIT is sensitive enough to identify subtle differences in dynamic sitting balance in individuals with various levels of balance impairment.
\end{abstract}

\section{Keywords}

Assessments, Barnett Balance Assessment-Sit, Balance, Normative Data

\section{Introduction}

Falls can impact individuals both mentally and physically. Falls themselves, as well as a fear of falling, can be attributed to both intrinsic and extrinsic factors. Intrinsic factors contributing to falls and a fear of falling include age-related physiological changes such as vision and hearing impairments, musculoskeletal decline, central nervous system (CNS) deterioration, and balance impairments. Additional intrinsic factors leading to falls can include cardiovascular, endocrine, neurological, and psychiatric disorders [1]. Extrinsic factors, including improper footwear or obstacles in the home, can affect balance and increase the fall risk among the elderly [1] [2]. Many of the balance and physiological deficits that are experienced after a fall emerge with age and can lead to decreased engagement in ADLs over time. 
In addition to contributing to decreased occupational engagement, falls also place a significant strain on the health care system. For example, a 2016 study conducted by Stevens, Sleet, Baldwin, and Noonan found that among individuals aged 65 years and older there were approximately 10,300 fatal falls and 2.6 million non-fatal fall related injuries among individuals in the United States. This same study found that direct medical costs for fatal falls totaled $\$ 2$ billion, and $\$ 19$ billion for non-fatal fall injuries. Of the $\$ 19$ billion for non-fatal injuries, $\$ 12$ billion was for hospitalization costs, $\$ 4$ billion for emergency department visits, and $\$ 3$ billion for outpatient treatment [3]. Implementation of effective and affordable preventative strategies may reduce the occurrence and cost of fall-related injuries. Thus, a reduction in fall incidences may result in a decrease in associated health care costs.

Falls do not always involve a loss of balance from a standing position. In 2010, an estimated 36,559 non-fatal wheelchair related accidents required emergency department attention. This statistic does not take into account falls that occurred when an individual was in a seated position or in a wheelchair. These accidents often occur when an individual is performing dynamic activities, such as reaching for objects at different heights, and experiences a fall. Falling from a seated position is, in particular, a problem for the elderly population, and should be addressed by healthcare professionals [4].

The Centers for Disease Control (CDC) has identified a need for improved comprehensive fall risk management programs that include fall prevention initiatives focusing on environmental and functional assessments [5]. Specifically, the CDC states that there is a need for occupational therapists to develop strategies to assess and evaluate fall risks because at this time "comprehensive multifactorial assessment and management interventions have not been clearly defined" ([5], p. 129). In the area of fall prevention, occupational therapy practitioners have a unique advantage in identifying barriers within the environment and developing assessments that mimic everyday environmental demands. Studies have suggested that there is a need to develop strategies to assist occupational therapists and their clients in collaboratively assessing fall risk [5].

When assessing clients with potential balance impairments, it is important to complete assessments in both the standing and seated position. Interestingly, the ability to remain stable while sitting requires functions similar to those required for functional mobility and gait. A study by Morgan [6] identified the relationship between sitting balance and gait, noting that the "assessment of static sitting balance in an acute stroke patient was found to be positively correlated with gait outcome" (p. 95). Gorman, Harro, and Platko [7] also highlighted the relationship between standing and sitting balance, stating "many scientists believe that concepts important for stance and postural control will be shown to be equally valid for postural control in sitting" (p. 4). Thus, it appears that establishing an understanding of functional sitting balance abilities among persons with limitations in standing should be an important component of the occupational therapy 
evaluation process.

There are currently a limited number of tools focusing specifically on the assessment of sitting balance. Previous tests designed specifically for assessment of sitting balance deficits include the Function in Sitting Test, the Sitting Balance Test, and the Ottawa Sitting Scale. However, the clinical usefulness of these tests is limited, due to unknown responsiveness of the tests and a lack of concurrent validity [7]. The Modified Functional Reach seated balance assessment is another tool currently utilized within clinical settings to assess sitting balance. However, this assessment only evaluates individuals when they are moving in one direction. Because many of the movements performed while seated occur along multiple planes of motion, it is important for clinicians to evaluate lateral reach in various planes during balance assessments [4].

The Barnett Balance Assessment-Sit (BBA-SIT) is a new assessment tool designed to allow clinicians to mimic and quantify various aspects of dynamic sitting balance during the completion of functional movements necessary to engage in meaningful activities from the seated position. It is based off of the original Barnett Balance Assessment (BBA), a standardized standing balance assessment tool that utilizes a Dynamic Arc to measure weight-shifting patterns during completion of reaching activities in various planes [8]. The apparatus consists of a moveable balance arc and arm, four eyelets, and four markers (see Figure 1). The BBA is administered while standing, in a series of three tests: the Ascending Reach, the Outward Reach, and the Balance Arc. Each of these three tests requires the client to complete "... reaching tasks involving various weight shifts in a specified pattern” ([9], p. 2). Clinicians can track a client's score in each of the three tests, as well as an overall combined score, over time to quantify changes in balance throughout the course of treatment [9].

One of unique features of the BBA is the fine-motor component of the evaluation. The BBA requires the client to move and place lightweight metal "markers" onto hooks in specific locations on the balance arc throughout the subtests (see Figure 1). Because the BBA requires the integration of gross- and fine-motor control with movements requiring dynamic weight shifting, the tool more closely mirrors daily activities than many currently-available balance assessments [9]. The BBA-SIT integrates these unique characteristics into an assessment designed to be completed while sitting.

While previous research has explored normative values of the BBA for standing balance with a non-balance impaired population [9], no research has yet established normative values for the BBA-SIT for sitting balance with a non-balance impaired population. Norms allow practitioners to identify the presence of performance deficits, by allowing for the comparison of a client's scores to those of a "typical" member of the population [10]. Without normative data for the BBA-SIT, a client's level or area of dysfunction in regard to sitting balance cannot be accurately assessed with the tool. The purpose of this descriptive quantitative study was to establish normative BBA-SIT values for non-balance 


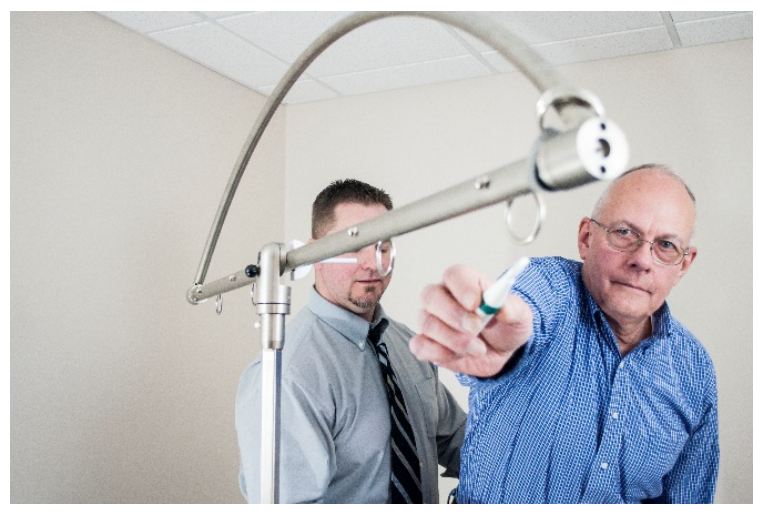

Figure 1. The dynamic arc. Image of patient reaching to place a marker on a hook of the balance arc.

impaired individuals in the following age groups: $18-29,30-39,40-49,50-$ $59,60-69,70+$.

\section{Method}

\section{Participants}

This research study utilized a quantitative descriptive approach to establish normative values for the BBA-SIT. The target population included healthy individuals who did not have a pre-existing balance deficit or orthopedic or neurologic condition that could impair balance. Convenience sampling and snowball sampling was used to recruit participants. Participants were recruited from community centers and neighborhoods in the researchers' areas of residence, and at the campus of the researchers' university (a medium-sized public university in the Midwestern United States).

To participate in the study, individuals were required to be 18 years or older, independently ambulatory without assistive devices, and able to follow simple verbal commands. Furthermore, they were required to be able to grasp and manipulate objects similar to those used in the BBA-SIT; sit independently without any assistance; and have no history of falls due to balance deficits within the past 12 months. A total of 180 people completed the study; this number was chosen based upon the researchers' time available for data gathering.

\section{Instrumentation}

A researcher-designed screening tool was utilized to determine if participants met criteria for inclusion in the study (as outlined in the previous paragraph). Standardized methods were used to set-up the BBA-SIT, and instructions for administering the assessment were utilized (refer to Appendix) to standardize the procedures followed by all four assessors. The BBA-SIT score sheet was utilized by all researchers to record the data.

\section{Apparatus}

The assessment tool for the BBA-SIT, the Dynamic Arc, consists of two components: the "arc" (the curved component) and the "arm" (the straight bar directly under the arc). These components are constructed from medical-grade metals 
and plastics [9]. Four eyelets are located on the underside of the arm; the client is asked to move markers on and off eyelets by completing arm movements within the transverse planes (Figure 2) and frontal planes (Figure 3). The design of the Dynamic Arc allows for the arm and arc to be moved into various positions to test participants' balance when reaching from different angles and distances [8].

Study site

Data collection occurred at various locations, including community centers and the researchers' university. These locations allowed for access to healthy individuals within multiple age groups. Data analysis occurred at the researchers' university.

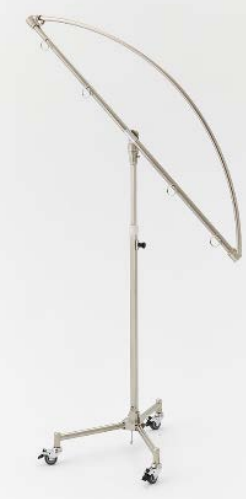

Figure 2. The dynamic arc in the transverse plane. Image shows the dynamic arc positioned in the transverse plane, one of the reaching patterns needed to complete the assessment.

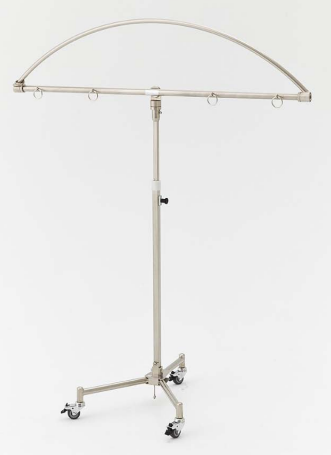

Figure 3. The dynamic arc in the frontal plane. Image shows the dynamic arc positioned in the frontal plane, one of the reaching patterns needed to complete the assessment. 


\section{Data collection}

The researchers' university granted IRB approval prior to initiation of the project. Prior to the start of data collection, the researchers were trained in the administration and the scoring of the BBA-SIT by the developer of the assessment.

During data collection, the researchers administered the assessment following the outline described within the BBA-SIT manual and BBA-SIT score sheet. Participants began the BBA-SIT in seated position, with feet flat on the floor, shoulder width apart; the upper body/trunk in a vertical position; and the shoulders vertically aligned with the hips. The assessment does not specify a specific chair height or type. One of the participant's arms was outstretched and positioned parallel to the floor (90 degrees of glenohumeral joint flexion), with their extended middle digit 3 inches away from the center of the BBA device [8]. Participants were asked to perform trunk rotation while performing specific reaching patterns to the left and right, one arm at a time, in order to physically manipulate BBA measurement markers (labeled A, B, C, or D) on the balance arm, in an order specified by the researcher (and outlined on the scoring sheet). Points were awarded for successful placement of measurement markers on the balance arm without a loss of balance ("no loss of balance" was defined in this assessment as the subject reaching out in order place the marker on the corresponding eyelet, and successfully returning to the original base of support while maintaining postural control throughout the process). The data was recorded on a standardized scoring sheet that was created specifically for the BBA-SIT.

\section{Data analysis}

Data was collected from a total of 180 participants and organized into the following mutually-exclusive age categories: 18 - 29, 30 - 39, 40 - 49, 50 - 59, 60 69 , and $70+$. In each of these age ranges, descriptive statistics were calculated using Microsoft Excel.

\section{Results}

A total of 180 people aged 18 - 92 met the inclusion criteria and participated in the study (see Table 1). No incentives were provided for study participation. All the study participants were recruited from one state in the Midwestern United States. The average age of participants was 49.6 years old (SD 18.7, range 74).

In each of the BBA subtests, no variation in scores was observed among the age categories. Each of the 180 participants scored the highest possible score (16 points), in each of the seven subtests, resulting in a perfect score of 112/112 for all participants. In each age category, all participants scored a perfect score.

\section{Discussion and Future Research Directions}

The purpose of this study was to collect quantitative data from a normal, non-impaired population to establish normative data for a new sitting balance assessment, the BBA-SIT. Data was collected from 180 non-balance impaired 
Table 1. Data for the Barnett Balance Assessment-SIT assessment in 180 study participants with no physical impairments.

\begin{tabular}{cccc}
\hline Age Group (years) & $n$ & Mean Age (SD) & Mean BBA-SIT Score \\
\hline $18-29$ & 30 & $22(3)$ & 112 \\
$30-39$ & 30 & $35(3)$ & 112 \\
$40-49$ & 30 & $46(3)$ & 112 \\
$50-59$ & 30 & $54(3)$ & 112 \\
$60-69$ & 30 & $64(3)$ & 112 \\
$70+$ & 30 & $75(22)$ & 112 \\
\hline
\end{tabular}

Note. Mean scores represent average age in years of the participants in the designated age group.

individuals ages 18 to $70+$. This study was the first to establish normative data for the BBA-SIT. The fact that all participants achieved a perfect score could potentially be the result of a ceiling effect in the BBA-SIT. It also alludes to a possible lack of discriminative power; the BBA-SIT may not be sensitive enough to detect important changes in balance in a non-impaired population or individuals with mild balance impairments, potentially limiting its usefulness in the clinical setting. Interestingly, research by Cercone et al. ([9], p. 1) on the BBA (designed to evaluate standing balance) found "little variation in total assessment scores in the age categories of $18-29,30-39$, and $40-49$, due to the presence of a ceiling effect." However, the researchers did find that "variations existed in scores among participants in the remaining age categories (50 - 59, $60-69$, and $70+)$." The researchers concluded that the BBA might lack discriminative power with populations that have no or mild balance impairments. It appears that similar issues might also be present in the BBA-SIT.

Further investigation of the clinical utility of the BBA-SIT is important for clinical purposes, as it is designed to evaluate sitting balance while individuals are completing dynamic reaching in various planes of motion. Current sitting balance assessments, including the Function in Sitting Test, the Sitting Balance Test, and the Ottawa Sitting Scale, do not assess an individual's balance in more than one plane of motion; their usefulness is limited due to unknown responsiveness and a lack of concurrent validity [7]. In particular, future research should utilize the BBA-SIT with a balance-impaired population, to determine if the test has discriminative power sufficient to detect the presence of small but important differences in sitting balance among individuals with various levels of balance impairments.

\section{Limitations}

One limitation of this study was the use of convenience sampling. All participants were recruited from one state in the Midwestern United States. Additionally, the sample demonstrated limited racial diversity, which may decrease generalizability of findings to the larger population. Furthermore, the researchers were only able to recruit 180 participants for the study, which is less than the 
amount included in many prior normative studies. Future studies of the BBA-SIT should utilize larger sample sizes, increasing the number of participants from 30 in each age group to 100. A final limitation of the study may have been inter-tester reliability. While all researchers were trained in the BBA-SIT administration and scoring protocol by its developer, subtle variations in administration could still have occurred between researchers.

\section{Conclusion}

This was the first normative study to provide data for the BBA-SIT, a new tool developed to evaluate sitting balance in individuals who are between the ages of 18 - 70+. The purpose of this descriptive study was to collect quantitative, normative data for each subtest of the BBA-SIT from individuals among six different age categories (18 - 29, 30 - 39, 40 - 49, 50 - 59, 60 - 69, and 70+). BBA-SIT scores were gathered from 180 participants who were determined to have no impairments in balance. The normative data collected showed no variation in scores across all six age categories. The lack of variation in scores suggests the potential presence of a ceiling effect, or that the BBA-SIT may not be sensitive enough to detect subtle but important balance deficits. Future research should be conducted to determine the ability of the BBA-SIT to detect deficits in sitting balance among a balance-impaired population, and if it has better discriminative capabilities with balance-impaired populations.

\section{References}

[1] Pasquetti, P., Apicella, L. and Mangone, G. (2014) Pathogenesis and Treatment of Falls in Elderly. Clinical Cases in Mineral and Bone Metabolism, 11, 222-225.

http://www.ncbi.nlm.nih.gov/pmc/articles/PMC4269147/ https://doi.org/10.11138/ccmbm/2014.11.3.222

[2] Oddsson, L.I.E., Boissy, P. and Melzer, I. (2007) How to Improve Gait and Balance Function in Elderly Individuals-Compliance with Principles of training. European Review of Aging and Physical Activity, 4, 15-23. https://doi.org/10.1007/s11556-007-0019-9

[3] Stevens, J.A., Sleet, D.A., Baldwin, G.T. and Noonan, R.K. (2016) Chapter 2: The Epidemiology and Risk Factors for Falls among Older Adults. In: Fall Prevention and Protection: Principles, Guidelines, and Practices, CRC Press, Boca Raton, FL, 19-28. https://doi.org/10.1201/9781315373744-3

[4] Thompson, M. and Medley, A. (2007) Forward and Lateral Sitting Functional Reach in Younger, Middle-Aged, and Older Adults. Journal of Geriatric Physical Therapy, 30, 43-48. https://doi.org/10.1519/00139143-200708000-00002

[5] Peterson, E.W., Finlayson, M., Elliott, S.J., Painter, J.A. and Clemson, L. (2012) Unprecedented Opportunities in Fall Prevention for Occupational Therapy Practitioners. American Journal of Occupational Therapy, 66, 127-130. https://doi.org/10.5014/ajot.2012.003814

[6] Morgan, P. (1994) The Relationship between Sitting Balance and Mobility Outcome in Stroke. Australian Journal of Physiotherapy, 40, 91-96.

http://ac.els-cdn.com/S0004951414604554/1-s2.0-S0004951414604554-main.pdf?_ti $\mathrm{d}=$ aaac03fc-0ba2-11e5-9fcd-00000aab0f02\&acdnat=1433523078_b1f41869853e74bd 
00d983ac62e430d0

https://doi.org/10.1016/S0004-9514(14)60455-4

[7] Gorman, S.L., Harro, C.C. and Platko, C. (2015) Don't Just Sit There: Evidence-Based Sitting Balance Examination \& Intervention.

http://c.ymcdn.com/sites/www.acutept.org/resource/resmgr/Don\%27t_Just_Sit_Th ere.pdf

[8] Functional-Innovation Enterprises (2013) The Barnett Rehabilitation System. https://functional-innovation.com/wp-content/uploads/2014/12/Manual-2014.pdf

[9] Cercone, M.L., Grulke-Kidd, K.M., Haskin, A.S., Medearis, K.M., Wegner, C.J. and Herlache-Pretzer, E. (2014) Establishing Normative Values for the Barnett Balance Assessment Tool: A Preliminary Study. The Open Journal of Occupational Therapy, 2, Article 5. https://doi.org/10.15453/2168-6408.1081

[10] Mitrushina, M.N., Boone, K.B., Razani, J. and D’Elia, L. (2005) Handbook of Normative Data for Neuropsychological Assessment. Oxford University Press, New York.

\section{Appendix}

Setup and Administration of the BBA-SIT Functional Sitting Balance Assessment

\section{REQUIREMENTS:}

- Sitting Surface (with/without back) which allows for the following positioning:

o Subject's feet flat on floor or hard surface

o Subject's knees in 90 degrees of flexion

o Subject's hips in 90 degrees of flexion

ADMINISTRATION

- Have subject properly positioned on sitting surface. Perform assessment in entirety, with scores recorded on BBA-SIT data sheets. Maintain safety with gait belt secured on subject throughout duration of assessment, with assessor maintaining stand-by assistance.

- No physical assistance is allowed unless required to maintain safety.

- All assessors are to be trained by the developer(s) of this assessment to ensure quality training and interrater reliability.

Position the Dynamic Arc in accordance to the data collection sheet. Each successfully placed marker is scored a point or a miss (pass/fail format). While sitting, the subject must reach out, place the marker on the corresponding eyelet, and successfully return to the original base of support while maintaining postural control throughout process. The scores are then tallied to determine the corresponding overall performance metric (N. Barnett, personal communication, June 20, 2016). 